\title{
Perturbative corrections to technicolor
}

\author{
A. C. Aguilar, ${ }^{1, *}$ A. Doff, ${ }^{2,3, \dagger}$ and A. A. Natale \\ ${ }^{1}$ University of Campinas-UNICAMP, Institute of Physics "Gleb Wataghin," \\ 13083-859 Campinas, São Paulo, Brazil \\ ${ }^{2}$ Universidade Tecnológica Federal do Paraná-UTFPR-DAFIS, \\ Avenida Monteiro Lobato Km 04, 84016-210 Ponta Grossa, Paraná, Brazil \\ ${ }^{3}$ Instituto de Física Teórica-UNESP, Rua Dr. Bento T. Ferraz, 271, \\ Bloco II, 01140-070 São Paulo, Brazil
}

(Received 9 February 2018; published 20 June 2018)

\begin{abstract}
The full solution of technicolor (TC) Schwinger-Dyson equations should include radiative corrections induced by extended technicolor (ETC) (or other) interactions. We verify that when TC is embedded into a larger theory including also QCD, these radiative corrections couple the different strongly interacting Schwinger-Dyson equations, providing a tiny mass to technifermions and changing the ultraviolet behavior of the gap equation solution. We argue about the origin of the different quark masses without appealing for different ETC boson masses, in one scenario where most of the new physics will appear in interactions with the third fermion generation and with a TC scalar boson possibly lighter than the TC characteristic scale $\left(\Lambda_{\mathrm{TC}}\right)$.
\end{abstract}

DOI: 10.1103/PhysRevD.97.115035

The origin of fermion and gauge boson masses in the standard model (SM) of elementary particles is explained by their interaction with the Higgs boson. The discovery of this boson at the LHC $[1,2]$ has crowned the SM; however, the data still cannot discard the possibility of this boson being a composite one. The case of a composite state, generating dynamical gauge symmetry breaking, instead of an elementary one, is more akin to the phenomenon of spontaneous symmetry breaking that originated from the Ginzburg-Landau Lagrangian. The latter can be derived from the microscopic Bardeen-cooper-schrieffer (BCS) theory of superconductivity describing the electron-hole interaction, which can be interpreted as a composite state. A similar mechanism happens in QCD where the chiral symmetry breaking is promoted by a nontrivial vacuum expectation value of a fermion bilinear operator and the Higgs role is played by the composite $\sigma$ meson. In particular, the technicolor (TC) idea was the earliest attempt to build models in this direction $[3,4]$.

The main ideas about TC models were reviewed in Refs. [5,6] and recent phenomenological studies about this class of models can be seen in Refs. [7-13] and references therein. Despite the fact that TC models are much more

\footnotetext{
*aguilar@ifi.unicamp.br †agomes@utfpr.edu.br ‡natale@ift.unesp.br
}

Published by the American Physical Society under the terms of the Creative Commons Attribution 4.0 International license. Further distribution of this work must maintain attribution to the author(s) and the published article's title, journal citation, and DOI. Funded by SCOAP ${ }^{3}$. complex than the ones with elementary scalar bosons, the main difficulty to build a realistic model lies in the ordinary behavior of the technifermion self-energy that is proportional to $\Sigma_{\mathrm{T}}\left(p^{2}\right) \propto \frac{\mu_{\mathrm{TC}}^{3}}{p^{2}}\left(p / \mu_{\mathrm{TC}}\right)^{\gamma}$ where $\mu_{\mathrm{TC}}$ is the characteristic TC dynamical mass at zero momentum and $\gamma$ the anomalous mass dimension. This self-energy leads to the known quark mass $\left(m_{\mathrm{Q}}\right)$ given by $m_{\mathrm{Q}} \propto \mu_{\mathrm{TC}}^{3} / M_{\mathrm{E}}^{2}$, where $M_{\mathrm{E}}$ is the mass of an extended technicolor boson (ETC), which is a particle that may change flavors. In order to describe, for example, the top quark mass we need a small $M_{\mathrm{E}}$ value, and this boson generates flavor changing neutral currents at one undesirable level. A possible solution to this dilemma requires a large $\gamma$ value [14], which can be obtained either with the introduction of (i) a large number of fermions or (ii) with a gauged four-fermion interaction [15-27]. Regardless of all these efforts, in these dynamical symmetry breaking models, it has not been clear up to now why the heaviest quark has a current mass of $\mathrm{O}(100) \mathrm{GeV}$ whereas the light quarks have a current mass of few $\mathrm{MeV}$. In addition, in the context of dynamical gauge symmetry breaking models, it is not naturally expected to have a scalar boson (the Higgs boson in this case) with a mass smaller than the Fermi or TC scale.

In this work, we argue that the answer to these questions may come out when QCD and TC are embedded into a larger group, possibly ETC or a grand unified theory (GUT). ${ }^{1}$ However there are two crucial requirements for

\footnotetext{
${ }^{1}$ One example of a model where TC and QCD are embedded into a larger ETC theory is the Farhi-Susskind GUT model [28].
} 


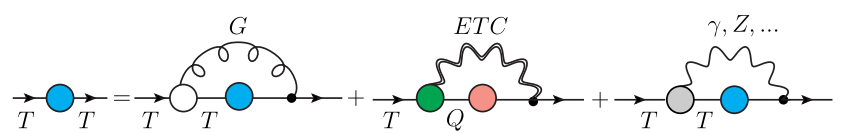

$\left(a_{1}\right)$

$\left(b_{1}\right)$

$\left(c_{1}\right)$

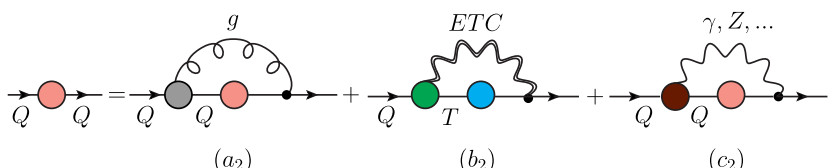

$\left(a_{2}\right)$

$\left(b_{2}\right)$

$\left(c_{2}\right)$

FIG. 1. The coupled system of SDEs for TC ( $\mathrm{T} \equiv$ technifermion $)$ and $\mathrm{QCD}(\mathrm{Q} \equiv$ quark) including ETC and electroweak or other corrections. $G(g)$ indicate technigluons (gluons).

this scenario to work out: (i) the interconnection of the Schwinger-Dyson equations (SDEs) describing the TC and QCD self-energies, as shown in Fig. 1, and (ii) the inclusion of a horizontal or family symmetry, where the third quark generation couples preferentially to TC and the first quark generation to QCD. As we see, a direct consequence of point (i) is the generation of a hard TC self-energy, which allows for a scalar boson mass smaller than $\Lambda_{\mathrm{TC}}{ }^{2}$.

The starting point in this analysis is the diagrammatic representation of the coupled SDEs for the techniquark and quark self-energies, shown in the first and second lines of Fig. 1, respectively. In this figure, the curly lines correspond to gluons (g) or technigluons $(\mathrm{G})$ and the wavy lines to the ETC (double) or other weakly interacting bosons. ${ }^{3}$

Notice that the above coupled system is rather intricate. More specifically, it involves different full boson propagators and fully dressed vertices, which should be closely intertwined through the different mass scales of the theories, namely, $\left(\Lambda_{\mathrm{QCD}, \mathrm{TC}, \mathrm{ETC}}\right)$. Here, we restrict ourselves to exploring the result of this coupled system in a rather simplified context. First, without specifying a model, we assume that QCD and TC are embedded into a gauge group, like the $S U(5)_{\mathrm{S}}$ of the Farhi-Susskind model. Then, we neglect the possible contributions that the diagrams $\left(c_{1}\right)$ and $\left(c_{2}\right)$ may give and later discuss their effects. Therefore, in our analysis, the conventional self-energies of the techniquarks $\left(a_{1}\right)$ and quarks $\left(a_{2}\right)$ only receive the perturbative corrections generated by the ETC interaction, which couples the techniquarks to quarks (and vice versa), as represented by the diagrams $\left(b_{1}\right)$ and $\left(b_{2}\right)$. Finally, we approximate the fully dressed propagators and vertices,

\footnotetext{
${ }^{2}$ Note that the ETC group can be composed either by TC and QCD as in the Farhi-Susskind model or it may include the electroweak group as well. As it is discussed later, the ETC role could even be played by a GUT that should include TC, QCD, and the electroweak theory. This is going to be possible because the fermion masses in our scenario are weakly dependent on the GUT mass.

${ }^{3}$ The SDE of Fig. 1 is similar to the ones describing the photon perturbative corrections to the quark mass [29], where it is understood that the strong interactions should be summed first [30].
}

entering into the gap equations, by their tree level expressions, ignoring the fact that the gauge bosons of the two strongly interacting theories are dynamically massive as suggested in a series of works [31-34].

After applying the above considerations, we arrive in the following coupled system of integral equations for the selfenergies of the techniquarks and quarks, respectively (in Euclidean space),

$\Sigma_{\mathrm{T}}\left(p^{2}\right)=3 \lambda_{\mathrm{T}} \int_{k} \frac{\Sigma_{\mathrm{T}}\left(k^{2}\right)}{(p-k)^{2}\left[k^{2}+\Sigma_{\mathrm{T}}^{2}\left(k^{2}\right)\right]}+m_{\mathrm{T}}\left(p^{2}\right)$,

$\Sigma_{\mathrm{Q}}\left(p^{2}\right)=3 \lambda_{\mathrm{Q}} \int_{k} \frac{\Sigma_{\mathrm{Q}}\left(k^{2}\right)}{(p-k)^{2}\left[k^{2}+\Sigma_{\mathrm{Q}}^{2}\left(k^{2}\right)\right]}+m_{\mathrm{Q}}\left(p^{2}\right)$,

with

$m_{\mathrm{T}(\mathrm{Q})}\left(p^{2}\right)=3 \lambda_{\mathrm{E}} \int_{k} \frac{\Sigma_{\mathrm{Q}(\mathrm{T})}\left(k^{2}\right)}{\left[(p-k)^{2}+M_{\mathrm{E}}^{2}\right]\left[k^{2}+\Sigma_{\mathrm{Q}(\mathrm{T})}^{2}\left(k^{2}\right)\right]}$,

where we have introduced the compact notation $\int_{k}=$ $1 /\left(2 \pi^{2}\right) \int_{0}^{\infty} d k^{2} k^{2} \int_{0}^{\pi} d \theta \sin ^{2} \theta$ with $\theta$ being the angle between the momenta $p$ and $k$. Moreover, we defined $\lambda_{i}=$ $C_{i} \alpha_{i}$ with $\mathrm{i}=\mathrm{T}, \mathrm{Q}$, and $\mathrm{E}$, where $\alpha_{i}$ are the TC, QCD, and ETC coupling constants, respectively. The $C_{i}$ are the corresponding Casimir eigenvalues for the different fermionic representations, i.e., $C_{\mathrm{Q}}=4 / 3, C_{\mathrm{T}}=3 / 4$, and $C_{\mathrm{E}}=1$, where this last value was chosen for simplicity, since we are not going to define one specific model for the ETC interaction.

We can easily identify the second term of Eq. (2) as an effective current quark mass obtained through TC interaction, described by diagram $\left(b_{2}\right)$. With the appropriate values for $\lambda_{\mathrm{Q}}, \lambda_{\mathrm{E}}$, and $M_{\mathrm{E}}$, we obtain a solution that is the sum of the dynamical quark mass with its effective current mass. In a similar way, Eq. (1) provides the dynamical techniquark mass with a very tiny effective current mass generated by the QCD correction, represented in the diagram $\left(b_{1}\right)$. If we perform a four-fermion approximation for the ETC contributions, by taking the limit of large $M_{\mathrm{E}}$ of the diagrams $\left(b_{1}\right)$ and $\left(b_{2}\right)$, the effective current masses added to the SDEs reduce to

$$
m_{\mathrm{T}(\mathrm{Q})} \propto \frac{\lambda_{\mathrm{E}}}{4 \pi M_{\mathrm{E}}^{2}} \int_{0}^{M_{\mathrm{E}}^{2}} d k^{2} \Sigma_{\mathrm{Q}(\mathrm{T})}\left(k^{2}\right)
$$

With the approximation performed in Eq. (4), which is equivalent to adding a bare mass, the solutions of Eqs. (1) and (2) are a superposition of the regular $\left[\propto 1 / p^{2}\right]$ plus irregular $\left[\propto \ln \left(p^{2}\right)^{-\gamma}\right]$ solutions [35-37]. Nowadays, it is known that the SDE solutions may vary between these two behaviors according to the boundary conditions [14-19,22-27,38-40], but we certainly can expect that a 
slowly decreasing with momentum self-energy dominates the large $p^{2}$ behavior.

We have solved numerically the coupled Eqs. (1) and (2) using $\alpha_{\mathrm{T}}=8.0$ and $\alpha_{\mathrm{Q}}=0.87$. The coupling values were chosen such that in isolation (i.e., $\alpha_{\mathrm{E}}=0$ ) the dynamical techniquark and quark masses generated are respectively $\mu_{\mathrm{TC}} \approx 1 \mathrm{TeV}$ and $\mu_{\mathrm{QCD}} \approx 0.3 \mathrm{GeV}$, and the solutions decrease as $1 / p^{2}$. When the ETC interaction is turned on, assuming $\alpha_{\mathrm{E}}=0.032$ and $M_{\mathrm{E}}=100 \mathrm{TeV}$, which was assumed as a typical ETC mass scale [5,28], we verified that the solutions seem to decrease like $1 / p^{2}$ after the $\mu_{\mathrm{TC}(\mathrm{QCD})}$ scale and appear to be basically flat at large momenta, consistent with a superposition of the regular and irregular solutions, although these large momenta were of the order of our numerical cutoff. This behavior is not unexpected, since the quark condensation gives a tiny mass to technifermions; however the asymptotic behavior, due to the current mass, is overwhelmed by the large dynamical TC mass, and it is difficult to extract a clear signal of the superposition of the different solutions from the full $\Sigma_{\mathrm{T}}\left(p^{2}\right)$ behavior.

Another way to verify that the self-energies decrease slowly with the momenta is to determine their anomalous dimension. As already mentioned, much of the information about chiral symmetry breaking resides in the boundary conditions of the SDE gap equation [38], from where we can derive the anomalous dimension, as shown in Ref. [40]. Using this observation, we determined an effective fourfermion coupling constant $\kappa_{\mathrm{E}} \propto C_{\mathrm{E}} \alpha_{\mathrm{E}} f\left(m_{\mathrm{T}}\left(M_{\mathrm{E}}\right), m_{\mathrm{Q}}\left(M_{\mathrm{E}}\right)\right)$ [41], and when this constant $\kappa_{\mathrm{E}}$ is introduced into Eq. (15) of Ref. [40], using $\mu_{\mathrm{TC}(\mathrm{QCD})}$ as the dynamical TC(QCD) masses of our coupled equations, we obtain $\gamma_{\mathrm{T}} \sim 2$. This indicates again a hard asymptotic behavior for $\Sigma_{\mathrm{T}}\left(p^{2}\right)$, corroborating the fact that the self-energies are changed when we consider the radiative corrections for the SDE; however, due to the approximations of Ref. [40], it is not possible to verify with high precision how the asymptotic behavior of $\Sigma_{\mathrm{T}}\left(p^{2}\right)$ and $\Sigma_{\mathrm{Q}}\left(p^{2}\right)$ are modified in the coupled system.

The best way to verify how the asymptotic behavior of $\Sigma_{\mathrm{T}}\left(p^{2}\right) \quad$ [or $\Sigma_{\mathrm{Q}}\left(p^{2}\right)$ ] has changed to an irregular-type solution is to compute the quark masses as a function of the ETC mass. This behavior is extremely dependent on the asymptotic self-energy. To observe this, let us suppose that the TC self-energy is

$$
\Sigma_{\mathrm{T}}\left(p^{2}\right) \approx \mu_{\mathrm{TC}}\left(\frac{\mu_{\mathrm{TC}}^{2}}{p^{2}+\mu_{\mathrm{TC}}^{2}}\right)
$$

which was conveniently normalized to the dynamical techniquark mass $\mu_{\mathrm{TC}}$ as $p^{2} \rightarrow 0$ and decays asymptotically as $1 / p^{2}$. Substituting Eq. (5) into Eq. (3), we obtain, in the limit of zero momentum, that

$$
m_{\mathrm{Q}} \propto \lambda_{E} \frac{\mu_{\mathrm{TC}}^{3}}{M_{\mathrm{E}}^{2}}
$$

On the other hand, if we assume that the TC self-energy is giving by an irregular-type solution, which can be cast in the form

$$
\Sigma_{\mathrm{T}}\left(p^{2}\right) \approx \mu_{\mathrm{TC}}\left[1+\delta_{1} \alpha_{T} \ln \left[\left(p^{2}+\mu_{\mathrm{TC}}^{2}\right) / \mu_{\mathrm{TC}}^{2}\right]\right]^{-\delta_{2}},
$$

where $\delta_{1}$ and $\delta_{2}$ are constants depending on the TC gauge group and fermionic representation. Note that Eq. (7) is also normalized to $\mu_{\mathrm{TC}}$ as $p^{2} \rightarrow 0$. It follows that the resulting quark mass calculated from Eq. (3) in this case is given by [42]

$$
m_{\mathrm{Q}} \propto \lambda_{E} \mu_{\mathrm{TC}}\left[1+\delta_{1} \alpha_{T} \ln \left(M_{\mathrm{E}}^{2} / \mu_{\mathrm{TC}}^{2}\right)\right]^{-\delta_{2}} .
$$

These quite different behaviors that may result for quark masses are one clear identifier of the asymptotic behavior of $\Sigma_{\mathrm{T}}\left(p^{2}\right)$ [and the same can be formulated with respect to $\left.\Sigma_{\mathrm{Q}}\left(p^{2}\right)\right]$. We stress that $m_{\mathrm{Q}}$ is the current mass at zero momentum, and the total mass should also include the dynamical mass and all momentum dependence.

Turning on the ETC interactions (by choosing either $\alpha_{\mathrm{E}}=0.032$ or $\alpha_{\mathrm{E}}=0.32$ ), we study numerically Eqs. (1) and (2) and determine how $\Sigma_{\mathrm{Q}}(0)$ behaves with $M_{\mathrm{E}}$ for the coupled SDE system. This behavior can be seen in Fig. 2. Because of the fact that the dynamical quark mass is negligible in comparison with the current mass, it turns out that $\Sigma_{\mathrm{Q}}(0) \cong m_{\mathrm{Q}}$. Then, $\Sigma_{\mathrm{Q}}(0)$ can be accurately fitted by

$$
m_{\mathrm{Q}}^{\mathrm{fit}}=a_{i}\left[\ln \left(M_{\mathrm{E}}^{2} / \mu_{\mathrm{TC}}^{2}\right)\right]^{-b_{i}},
$$

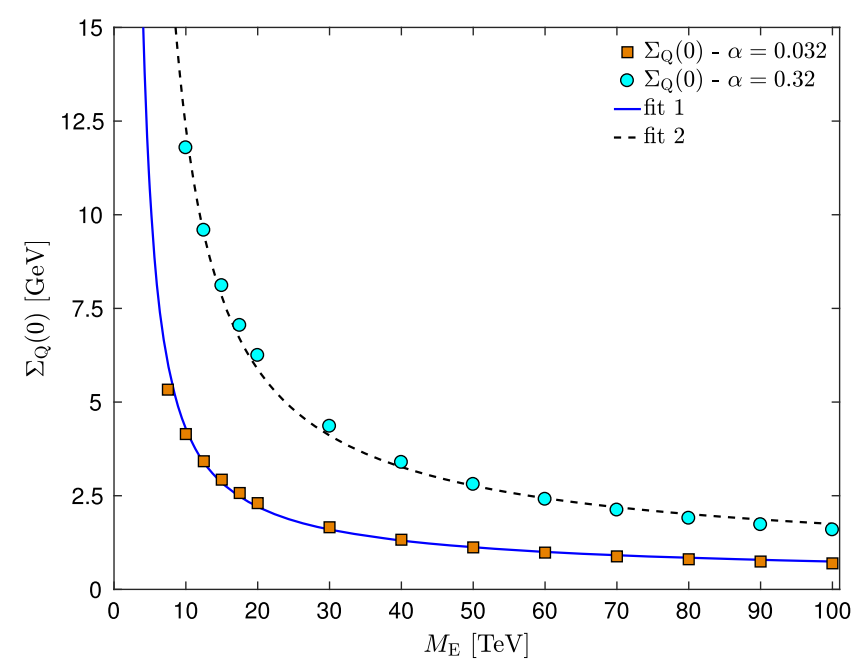

FIG. 2. The comparison of the behavior of $\Sigma_{\mathrm{Q}}(0)$ as a function of $M_{\mathrm{E}}$ with the fit given by Eq. (9). Fit 1 was obtained with $\alpha_{\mathrm{E}}=0.032$ and fit 2 with $\alpha_{\mathrm{E}}=0.32$; the other parameters are described in the text. 
which is precisely the same behavior found in Eq. (8). More specifically, the set of optimal values when $\alpha_{\mathrm{E}}=0.032$ is $a_{1}=203.92 \mathrm{GeV}$ and $b_{1}=2.53$, to be denoted by fit 1 . For fit 2, defined when $\alpha_{\mathrm{E}}=0.32$, we have $a_{2}=912.9 \mathrm{GeV}$ and $b_{2}=2.82$. The reduced chi square of both fits is $R^{2}=0.99$. The quark mass turns out to be heavy and of order of a few $\mathrm{GeV}$ as can be seen in Fig. 2. However, we have to remember that in the numerical calculation, we used $C_{\mathrm{E}}=1$, which is a rather naive assumption, since any theory embedding TC and QCD is represented by a large gauge group with fermions in higher dimensional representations and increased $C_{\mathrm{E}}$ values. Moreover, as in the GUT model of Ref. [28], one specific quark may obtain mass from different diagrams classified as $\left(b_{2}\right)$ in Fig. 1, which when added generate masses up to $\mathrm{O}(100) \mathrm{GeV}$. These masses are roughly proportional to $\mu_{\mathrm{TC}}$ [of $\left.\mathrm{O}(1) \mathrm{TeV}\right]$ times $\lambda_{E}$.

The fit of Eq. (9) shows unmistakably how the logarithmic factor enters into action in Fig. 2, and is consistent with the prediction of Eq. (8). It must be remembered that $m_{\mathrm{Q}}$, given by Eq. (3), also runs and the full result of the quark mass should include the momentum dependence. The large coefficients $a_{i}$ appearing in Eq. (9) are just a consequence of extending our fit, which is only appropriate for the asymptotic regime of the dynamical masses, to small $M_{\mathrm{E}}$ values. We recall that such small $M_{\mathrm{E}}$ values are not phenomenologically acceptable mass scales, if this theory is supposed to embed TC and QCD. We also do not expect that technigluon (gluon) masses and vertex corrections affect these results. Notice that in this scenario it is perfectly possible to obtain an infrared mass of $\mathrm{O}(100) \mathrm{GeV}$, which is of the order of the top quark mass.

The coupled SDE system indicates that the techniquarks obtain a dynamical mass $\left(\mu_{\mathrm{TC}}\right)$ that at zero momentum is of order of $1 \mathrm{TeV}$. The self-energy momentum dependence is a superposition of the regular and irregular solution, whose asymptotic behavior is dominated by the irregular solution that appears due to a tiny current mass generated by the QCD condensation. Quark masses also have a dynamical mass of the order of $300 \mathrm{MeV}$; however, they can obtain a current mass from TC condensation up to $\mathrm{O}(100) \mathrm{GeV}$, which can explain the third generation quark masses. In this case, the total quark mass is totally dominated by the irregular solution, i.e., the one that runs with the momentum as a logarithm. Apart from the small logarithmic dependence on $M_{\mathrm{E}}$, the ordinary quark masses are always proportional to $\alpha_{\mathrm{E}} C_{\mathrm{E}} \mu_{\mathrm{TC}}$, as given by Eq. (8). The technifermion masses are mostly dynamical and proportional to $\mu_{\mathrm{TC}}$. However this scenario is not complete until we take into account all radiative corrections. We have neglected the diagrams $\left(c_{1}\right)$ and $\left(c_{2}\right)$ of Fig. 1. All selfenergies in these diagrams also run logarithmically with the momentum, and the diagram $\left(c_{1}\right)$ generates for the techniquarks an effective mass proportional to $\mu_{\mathrm{TC}}$ times, for example, an electroweak charge or any other charge of interaction that contributes to the third diagram of Fig. 1, and, depending on the model, an ETC charge. Ordinary quarks also obtain a mass of a few $\mathrm{MeV}$, which may appear, due to the electroweak or other interactions, depicted in the diagram $\left(c_{2}\right)$. For instance, for the QED interaction we have $m_{\mathrm{Q}} \propto \alpha_{e m} e_{q}^{2} \mu_{\mathrm{QCD}}$, where $\alpha_{e m}$ is the QED coupling constant and $e_{q}$ the quark charge. This fact leads to a quite interesting solution for the fermionic mass spectra. In this scenario we must have a family or horizontal symmetry imposing that the third quark family couples preferentially to TC, i.e., the diagram $\left(b_{2}\right)$ of Fig. 1, whereas the first quark family obtains masses preferentially from QCD, receiving most of the radiative contributions from the diagram $\left(c_{2}\right)$. The final quark mass matrix can be of the Fritzch type

$$
m_{f}=\left(\begin{array}{ccc}
0 & A & 0 \\
A^{*} & 0 & B \\
0 & B^{*} & C
\end{array}\right),
$$

where $A \approx \alpha_{e m} e_{q}^{2} \mu_{\mathrm{QCD}}$ and $C \approx \alpha_{\mathrm{E}} C_{\mathrm{E}} \mu_{\mathrm{TC}}$, providing a natural explanation of the different mass scales. The factor $B$ should also appear naturally and be between $A$ and $C$, because the TC and QCD scalars mix, due to the many interactions that may connect QCD and TC.

To produce a mass matrix like the one of Eq. (10) we can choose as a horizontal symmetry the $S U(3)_{\mathrm{H}}$ group assigning to the first and third family different quantum numbers, in such a way that the third family couples only to TC and the first one only to QCD. Higher order loop contributions to the SDE give intermediate masses to the second quark generation, which explains the origin of the term $B$.

To verify the origin of the term $B$, let us represent the TC and QCD scalar composite fields by $\eta$ and $\phi$ that will be formed in the $\overline{\mathbf{6}}$ and $\mathbf{3}$ representations of $S U(3)_{\mathrm{H}}$. The most general effective potential is described by

$V(\eta, \phi)=\mu_{\eta}^{2} \eta^{\dagger} \eta+\lambda_{\eta}\left(\eta^{\dagger} \eta\right)^{2}+\mu_{\phi}^{2} \phi^{\dagger} \phi+\lambda_{\phi}\left(\phi^{\dagger} \phi\right)^{2}$,

where we can identify the vacuum expectation values (vevs) of the TC and QCD condensates as given by the ratio of their respective masses and couplings, i.e.,

$$
v_{\eta}^{2}=-\frac{\mu_{\eta}^{2}}{\lambda_{\eta}}, \quad v_{\phi}^{2}=-\frac{\mu_{\phi}^{2}}{\lambda_{\phi}} .
$$

Such potential is quite plausible if we consider the results of Refs. $[43,44]$, where it was shown that the interactions of a composite Higgs boson are very similar to the ones of a fundamental boson. This system leads to an intermediate mass scale and to a mass matrix identical to Eq. (10).

The QCD and TC vevs, due to the horizontal symmetry, can be written respectively in the following form $[45,46]$, 


$$
\langle\eta\rangle \sim\left(\begin{array}{c}
0 \\
0 \\
v_{\eta}
\end{array}\right), \quad\langle\phi\rangle \sim\left(\begin{array}{ccc}
0 & 0 & 0 \\
0 & 0 & 0 \\
0 & 0 & v_{\phi}
\end{array}\right),
$$

which are of the order of approximately $250 \mathrm{MeV}$ and $250 \mathrm{GeV}$. We can now verify what fermionic mass matrix one can obtain with the vevs of Eq. (13). Assuming that the composite scalars $\eta$ and $\phi$ have ordinary Yukawa couplings to fermions described by the following effective Yukawa Lagrangian,

$$
\mathcal{L}_{Y}=a \bar{\Psi}_{L \lambda}^{i} \eta_{\lambda}^{k} U_{R}^{j} \epsilon_{i j k}+b \bar{\Psi}_{L \lambda}^{i} \phi^{i j} U_{R}^{j},
$$

where $\Psi$ and $U$ are the ordinary fermion fields. In addition, $\lambda$ is a weak hypercharge $S U(2)_{w}$ index. For instance, $\lambda=1$ represents charge $2 / 3$ quarks and $\lambda=2$ correspond to the charge $1 / 3$ quarks. In addition, $i, j$, and $k$ indicate the components of the composite scalar bosons of the representations $\mathbf{3}$ and $\overline{\mathbf{6}}$ of $S U(3)_{H} ; a$ and $b$ are the coupling constants. Substituting the vevs of Eq. (13) in the Yukawa Lagrangian for the charge $2 / 3$ quarks, we obtain

$$
\mathcal{L}_{Y}=a \bar{c}_{L} v_{\eta} u_{R}-a \bar{u}_{L} v_{\eta} c_{R}+b \bar{t}_{L} v_{\varphi} t_{R}
$$

leading to a mass matrix in the $(u, c, t)$ basis that is given by

$$
\bar{m}^{\frac{2}{3}}=\left(\begin{array}{ccc}
0 & -a v_{\eta} & 0 \\
a v_{\eta} & 0 & 0 \\
0 & 0 & b v_{\phi}
\end{array}\right) .
$$

The third generation fermions obtain large masses because coupling directly to technifermions, while the first generation ones obtain masses coupling to ordinary quarks. Having this picture in mind, we can now see that the most general vev for this system includes the mass generation for the intermediate family.

Note that there is no way to prevent the coupling at higher order of the different composite scalar bosons with $S U(3)_{H}$ quantum numbers. Examples of such couplings are shown in Fig. 3, where the effective coupling between scalars and gauge bosons involves the self-energy solution that we have discussed, and is also enhanced due to its hard behavior with the momentum.

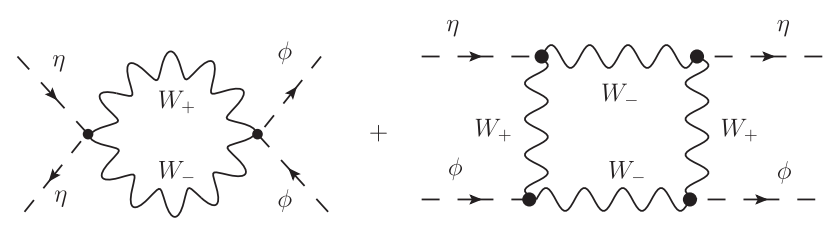

FIG. 3. Higher order corrections coupling the $\eta$ and $\phi$ composite bosons. The effective coupling between two scalars and two W's occurs through a fermion loop.
These diagrams produce the following contribution to the potential,

$$
V_{\delta}(\eta, \phi)=\lambda_{1} \eta^{\dagger} \eta \phi^{\dagger} \phi+\lambda_{2} \eta^{\dagger} \phi \eta \phi^{\dagger}+\cdots,
$$

which should be added to Eq. (11), shifting the vev matrix in order to reproduce a fermionic Fritzch mass matrix of Eq. (10). Details about this mechanism can be seen in Refs. [45-47].

Therefore, the mass generation of the different fermion families is just one effect of the alignment of the two different strong interactions in the presence of the horizontal symmetry. Another type of model, which naturally suppresses the TC coupling to the light quarks, can be formulated choosing the technifermion representation under QCD in such a way that they do not couple, at leading order, to the first two quark generations.

Up to now we have not discussed the leptonic mass spectra. We say that the family or horizontal symmetry should also be extended to the leptonic sector. In this case only the $\tau$ lepton obtains mass from technifermion condensation, and the other first two leptonic generations remain massless. More complicated mass spectra depend on specific models. In order to give an idea of the possible different models, let us now indicate techniquarks and technileptons by $\mathrm{T}$ and $\mathrm{L}$, whereas ordinary quarks and leptons are indicated by $Q$ and $\ell$, respectively, and assume that there is a family symmetry imposing that the TC sector connects only to the third ordinary fermion generation. If technileptons couple only to themselves and to ordinary leptons (i.e., there is not LT or LQ coupling) the technilepton self-energy is decoupled from the others, and its solution is soft $\left(\propto 1 / p^{2}\right)$. In this case only the $\tau$ lepton obtains a tiny mass. If we now admit that the theory has LT and/or LQ couplings, then we have a new set of coupled equations. Now the technilepton self-energy is hard, as in the techniquark case, and the $\tau$ lepton may obtain a few $\mathrm{GeV}$ mass. Finally, a leptonic mass matrix like the one of Eq. (10) is generated only at higher perturbative level, when ordinary quarks and leptons may be unified at a deeper level (with, for instance, $\ell \mathrm{Q}$ couplings), and probably related to some very heavy unified gauge boson mass. Generating naturally $\ell$ masses smaller than $\mathrm{Q}$ masses. Note that the larger the gauge group is unifying these theories the more complex the set of coupled SDE. Of course, here we are assuming only left-handed neutrinos, and a full explanation of the fermionic mass spectra (including neutrino masses) is a hard task and out of the scope of this work.

It is interesting to give an idea of what kind of model it is possible to build in this scenario. We can follow similar ideas as the ones in the Farhi-Susskind model. In that model, the $S U(5)_{\mathrm{S}}$ group was broken to $S U(2)_{\mathrm{HC}}$ and $S U(3)_{c}$, where $H C$ indicates the hypercolor (or TC) theory; however it was not discussed in detail how the $S U(2)$ scale $\Lambda_{\mathrm{HC}}$ could be larger than $\Lambda_{\mathrm{QCD}}$. In principle, we can adopt 
the most attractive channel hypothesis to have one idea about the symmetry breaking of the larger group. It is not impossible that the symmetry breaking pattern of a quite large group leads to a group smaller than $S U(3)_{c}$ with a fundamental scale larger than $\Lambda_{\mathrm{QCD}}$, since this is a quite model dependent problem. However, the most probable breaking of a larger group into QCD plus another stronger interaction with $\Lambda_{\mathrm{TC}}>\Lambda_{\mathrm{QCD}}$ would happen when TC is given at least by a $S U(4)_{\mathrm{TC}}$ group.

Now, let us consider a unified theory based on the $S U(9)$ gauge group, containing a $S U(4)_{\mathrm{TC}} \mathrm{TC}$ theory and the standard model. The anomaly free fermionic representations of this theory are [48]

$$
5 \otimes[9,8]_{i} \oplus 1 \otimes[9,2]_{i},
$$

where $i=1,2,3$ is a family or horizontal index that appears due to the necessary replication of families associated to a $S U(3)_{\mathrm{H}}$ horizontal group; the [ $\left.\underline{8}\right]$ and [2] are antisymmetric under $S U(9)$. These representations can be decomposed according to the group product $S U(4)_{\mathrm{TC}} \otimes S U(5)_{g g}$, where $S U(5)_{g g}$ is the standard Georgi-Glashow GUT [49]. The technifermions in this model transforming as $[4,5]_{i}$ and $[\overline{4}, 1]_{i}$ should have different quantum $S U(3)_{\mathrm{H}}$ numbers than the ordinary fermions transforming as $[1,10]_{i}$ and $[\overline{6}, 1]_{i}$ in order to produce a matrix like Eq. (10). According to the most attractive channel hypothesis [50,51], for the TC and QCD condensates (and their scalar bosons) appearing respectively in the $\overline{\mathbf{6}}$ and $\mathbf{3}$ of the $S U(3)_{\mathrm{H}}$, as discussed previously, it is enough that the standard left-handed (righthanded) fermions transform as triplets (antitriplets) under $S U(3)_{\mathrm{H}}$. Evidently, the full set of coupled SDE in this specific model is extremely complex involving couplings of the $S U(9), S U(5)_{g g}$, SM ones, and the horizontal bosons, and all their implications are analyzed in a future work.

We may wonder what happens with pseudo-Goldstone bosons in the scenario we are proposing here. Diagram $\left(a_{1}\right)$ of Fig. 1 generates a dynamical TC mass at a TeV scale. However, assuming that technifermions have an electroweak or other similar charge, the diagrams $\left(b_{1}\right)$ and $\left(c_{1}\right)$ of Fig. 1 generate effective "bare" masses $m_{T} \propto \alpha_{i} \mu_{T C} \propto$ $O(1-10) \mathrm{GeV}$. In this case, we can obtain a lower bound on the pseudo-Goldstone masses $\left(m_{\Pi}\right)$ using the Gell-MannOakes-Renner relation to estimate $m_{\Pi}^{2} \approx m_{T}\left\langle\bar{\psi}_{T} \psi_{T}\right\rangle / 2 F_{\Pi}^{2}$, where in the right-hand side we have the TC condensate divided by the technipion decay constant. This relation roughly implies a lower bound of $O(30-90) \mathrm{GeV}$ for the pseudo-Goldstone masses.

Another way to observe the increase of the pseudoGoldstone masses is to compute the effect of electroweak (or other) radiative corrections to these bosons. The diagram of Fig. 4 shows the radiative correction to the pseudo-Goldstone boson mass induced by one gauge boson A with coupling constant $g_{A}$, mass $M_{A}$, and a vertex

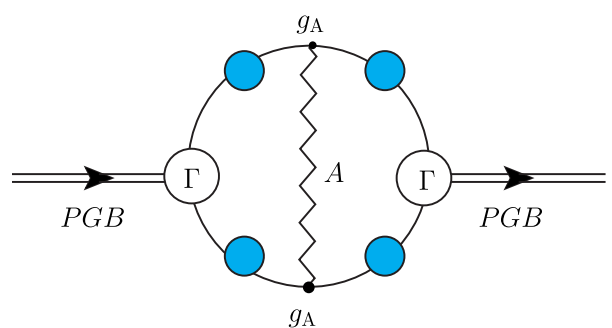

FIG. 4. Radiative correction to the mass of the pseudo-Goldstone boson.

indicated by $\Gamma$ that is proportional to the technipion wave function. It is quite important to remember that the technipion wave function is related to the technifermion self-energy as $\left.\Phi_{B S}^{\Pi}(p, q)\right|_{q \rightarrow 0} \approx \Sigma_{T}\left(p^{2}\right)$. Therefore we can recognize that the calculation of this diagram is quite different if the technipion wave function (or the vertex) is hard or soft. A rough evaluation of this diagram within the dynamical perturbation theory approach [52] gives

$$
m_{\Pi}^{2} \propto g_{A}^{2}\left(\frac{\mu_{\mathrm{TC}}^{2}}{F_{\Pi}^{2}}\right) M_{A}^{2}
$$

This calculation is certainly very model dependent. However, when the pseudoscalar vertex is soft the result turns out to be suppressed by the $M_{A}$ mass, and does not increase with $M_{A}$ as shown in Eq. (19). As a consequence pseudo-Goldstone boson masses turn out to be heavier in the scenario presented here.

Finally a last consequence that results from the slowly decreasing TC self-energy is the possible explanation of why the observed Higgs boson mass $\left(m_{H}\right)$ may be so light compared with the composition mass scale $\left(\mu_{\mathrm{TC}}\right)$. The conventional prediction for the scalar mass in composite models is $m_{H}^{2} \approx 4 \mu_{\mathrm{TC}}^{2}$, derived from the homogeneous Bethe-Salpeter equation (BSE). However, this relation is modified by the inhomogeneous BSE normalization condition when the scalar wave function, which is directly related to the fermion self-energy, is a slowly decreasing function with the momentum. In this case, the above mass relation is then replaced by $m_{H}^{2} \approx 4 \mu_{\mathrm{TC}}^{2} f\left(\alpha_{\mathrm{T}}, C_{\mathrm{T}}\right)$ where the function $f\left(\alpha_{\mathrm{T}}, C_{\mathrm{T}}\right)$ depends on the TC gauge group, its coupling constant and fermionic representation, and can be naturally a factor of $O(1 / 10)$ [53-55].

In this work we have given evidences that radiative corrections to TC (QCD) change the UV technifermion (quark) self-energy behavior. This happens when TC and QCD are embedded in a unified theory as in the FarhiSusskind model. This fact can be observed by noticing that there are perturbative contributions to the SDE that introduce effective four-fermion interactions like the one shown in Eq. (4), or by modifications of the gap equation boundary conditions that produce a large mass anomalous dimension. However the most clear evidence of a hard 
asymptotic self-energy is shown in Fig. 2, which indicates generated current masses weakly dependent on the ETC boson mass. As a consequence a large splitting between the masses of different generations must appear by the imposition of a family or horizontal symmetry in such a way that TC gives masses preferentially to the third family and QCD to the first one. Of course, the horizontal or family symmetry also generates an enormous complexity to the coupled set of gap equations, which is peculiar to the attempt of obtaining the fermion mass spectra in the context of dynamical symmetry breaking, but the origin of these mass spectra is just a consequence of the two strong interactions aligning with the horizontal symmetry. The experimental consequences of this scenario are that the new interactions with TC, which can be simply based on one $S U(2)_{\mathrm{TC}}$ theory, mostly occur with the third fermionic generation; and the scalar boson mass, the one playing the role of the Higgs boson, can also be lighter than usually thought. The scalar boson mass $\left(m_{H}\right)$ relation with $\mu_{\mathrm{TC}}$ is modified by the BSE normalization condition, which suppresses the ordinary expected value $m_{H}^{2} \approx 4 \mu_{\mathrm{TC}}^{2}$. In this type of model it is possible that TC pseudo-Goldstone boson masses may also turn out to be large and consistent with the present experimental limits [42,47], and all these aspects are under study [41].

\section{ACKNOWLEDGMENTS}

This research was partially supported by the Conselho Nacional de Desenvolvimento Científico e Tecnológico (CNPq) under Grants No. 305815/2015 (A.C. A.), No. 302663/2016-9 (A. D.), and No. 302884/2014 (A. A. N.), and by Grants No. 2013/22079-8 (A. A. N.), No. 2017/07595, and No. 2017/05685-2 (A. C. A.) of Fundação de Amparo à Pesquisa do Estado de São Paulo (FAPESP).
[1] G. Aad et al. (ATLAS Collaboration), Phys. Lett. B 716, 1 (2012).

[2] S. Chatrchyan et al. (CMS Collaboration), Phys. Lett. B 716, 30 (2012).

[3] S. Weinberg, Phys. Rev. D 19, 1277 (1979).

[4] L. Susskind, Phys. Rev. D 20, 2619 (1979).

[5] E. Farhi and L. Susskind, Phys. Rep. 74, 277 (1981).

[6] V. A. Miransky, Dynamical Symmetry Breaking in Quantum Field Theories (World Scientific Co., Singapore, 1993).

[7] J. R. Andersen et al., Eur. Phys. J. Plus 126, 81 (2011).

[8] F. Sannino, J. Phys. Conf. Ser. 259, 012003 (2010).

[9] F. Sannino, arXiv:1306.6346.

[10] F. Sannino, Int. J. Mod. Phys. A 25, 5145 (2010).

[11] F. Sannino, arXiv:0911.0931

[12] F. Sannino, Int. J. Mod. Phys. A 20, 6133 (2005).

[13] A. Belyaev, M. S. Brown, R. Foadi, and M. T. Frandsen, Phys. Rev. D 90, 035012 (2014).

[14] B. Holdom, Phys. Rev. D 24, 1441 (1981).

[15] K. D. Lane and M. V. Ramana, Phys. Rev. D 44, 2678 (1991).

[16] T. W. Appelquist, J. Terning, and L. C. R. Wijewardhana, Phys. Rev. Lett. 79, 2767 (1997).

[17] K. Yamawaki, Prog. Theor. Phys. Suppl. 180, 1 (2010); arXiv:9603293.

[18] Y. Aoki, T. Aoyama, M. Kurachi, T. Maskawa, K.-i. Nagai, H. Ohki, A. Shibata, K. Yamawaki, and T. Yamazaki (LatKMI Collaboration), Phys. Rev. D 85, 074502 (2012).

[19] T. Appelquist, K. Lane, and U. Mahanta, Phys. Rev. Lett. 61, 1553 (1988).

[20] R. Shrock, Phys. Rev. D 89, 045019 (2014).

[21] M. Kurachi and R. Shrock, J. High Energy Phys. 12 (2006) 034.

[22] V. A. Miransky and K. Yamawaki, Mod. Phys. Lett. A 04, 129 (1989).
[23] K.-I. Kondo, H. Mino, and K. Yamawaki, Phys. Rev. D 39, 2430 (1989).

[24] V. A. Miransky, T. Nonoyama, and K. Yamawaki, Mod. Phys. Lett. A 04, 1409 (1989).

[25] T. Nonoyama, T. B. Suzuki, and K. Yamawaki, Prog. Theor. Phys. 81, 1238 (1989).

[26] V. A. Miransky, M. Tanabashi, and K. Yamawaki, Phys. Lett. B 221, 177 (1989).

[27] K.-I. Kondo, M. Tanabashi, and K. Yamawaki, Mod. Phys. Lett. A 08, 2859 (1993).

[28] E. Farhi and L. Susskind, Phys. Rev. D 20, 3404 (1979).

[29] S. J. Brodsky, G. F. de Téramond, and I. A. Schmidt, Phys. Rev. Lett. 44, 557 (1980).

[30] N. S. Craigie, S. Narison, and Riazuddin, Nucl. Phys. B174, 207 (1980).

[31] J. M. Cornwall, Phys. Rev. D 26, 1453 (1982).

[32] A. C. Aguilar, D. Binosi, and J. Papavassiliou, Phys. Rev. D 78, 025010 (2008).

[33] A. C. Aguilar and J. Papavassiliou, Phys. Rev. D 83, 014013 (2011).

[34] A. Doff, F. A. Machado, and A. A. Natale, Ann. Phys. (Amsterdam) 327, 1030 (2012).

[35] K. Lane, Phys. Rev. D 10, 2605 (1974).

[36] P. Langacker, Phys. Rev. Lett. 34, 1592 (1975).

[37] H. Pagels, Phys. Rev. D 21, 2336 (1980).

[38] A. Cohen and H. Georgi, Nucl. Phys. B314, 7 (1989).

[39] T. Takeuchi, Phys. Rev. D 40, 2697 (1989).

[40] A. Doff and A. A. Natale, Phys. Lett. B 771, 392 (2017).

[41] A. Doff and A. A. Natale, arXiv:1803.10458.

[42] A. A. Natale, Z. Phys. C 21, 273 (1984).

[43] J. D. Carpenter, R. E. Norton, and A. Soni, Phys. Lett. B 212, 63 (1988).

[44] J. Carpenter, R. Norton, S. Siegemund-Broka, and A. Soni, Phys. Rev. Lett. 65, 153 (1990). 
[45] F. Wilczek and A. Zee, Phys. Rev. Lett. 42, 421 (1979).

[46] G. B. Gelmini, J. M. Gerard, T. Yanagida, and G. Zoupanos, Phys. Lett. B 135, 103 (1984).

[47] A. Doff and A. A. Natale, Eur. Phys. J. C 32, 417 (2003).

[48] P. H. Frampton, Phys. Rev. Lett. 43, 1912 (1979); 44, 299(E) (1980).

[49] H. Georgi and S. L. Glashow, Phys. Rev. Lett. 32, 438 (1974).

[50] J. M. Cornwall, Phys. Rev. D 10, 500 (1974).
[51] S. Raby, S. Dimopoulos, and L. Susskind, Nucl. Phys. B169, 373 (1980).

[52] H. Pagels and S. Stokar, Phys. Rev. D 20, 2947 (1979).

[53] A. Doff, A. A. Natale, and P. S. Rodrigues da Silva, Phys. Rev. D 80, 055005 (2009).

[54] A. Doff and A. A. Natale, Int. J. Mod. Phys. A 31, 1650024 (2016).

[55] A. Doff, A. A. Natale, and P. S. Rodrigues da Silva, Phys. Rev. D 77, 075012 (2008). 